\title{
Immunocytes as a Biocarrier to Delivery Therapeutic and Imaging Contrast Agents to Tumors
}

\author{
Jinhyang Choi, ${ }^{1}$ Ha-Na Woo, ${ }^{1}$ Eun Jin Ju, ${ }^{1}$ Joohee Jung, ${ }^{1,2}$ Hye-Kyung Chung, ${ }^{3}$ \\ Jaesook Park, ${ }^{1}$ Seok Soon Park, ${ }^{1}$ Seol Hwa Shin, ${ }^{3}$ Hye Ji Park, ${ }^{3}$ Jin Seong Lee, ${ }^{4}$ \\ Si Yeol Song, ${ }^{1,5}$ Seong-Yun Jeong, ${ }^{1}$ and Eun Kyung Choi ${ }^{1,3,5}$ \\ ${ }^{1}$ Institute for Innovative Cancer Research, Asan Medical Center, University of Ulsan College of Medicine, \\ Seoul 138-736, Republic of Korea \\ ${ }^{2}$ College of Pharmacy, Duksung Women's University, Seoul 132-714, Republic of Korea \\ ${ }^{3}$ Center for Development and Commercialization of Anti-Cancer Therapeutics, Asan Medical Center, Seoul 138-736, Republic of Korea \\ ${ }^{4}$ Department of Radiology and Research Institute of Radiology, Asan Medical Center, University of Ulsan College of Medicine, \\ Seoul 138-736, Republic of Korea \\ ${ }^{5}$ Department of Radiation Oncology, Asan Medical Center, University of Ulsan College of Medicine, Seoul 138-736, Republic of Korea
}

Correspondence should be addressed to Seong-Yun Jeong, syj@amc.seoul.kr and Eun Kyung Choi, ekchoi@amc.seoul.kr

Received 13 February 2012; Revised 10 May 2012; Accepted 13 May 2012

Academic Editor: Patricia Murray

Copyright (c) 2012 Jinhyang Choi et al. This is an open access article distributed under the Creative Commons Attribution License, which permits unrestricted use, distribution, and reproduction in any medium, provided the original work is properly cited.

\begin{abstract}
Radiotherapy for cancer treatment has been used for primary or adjuvant treatment in many types of cancer, and approximately half of all cancer patients are undergoing radiation. However, ionizing radiation exposure induces genetic alterations in cancer cells and results in recruitment of monocytes/macrophages by triggering signals released from these cells. Using this characteristic of monocytes/macrophages, we have attempted to develop a biocarrier loading radiosensitizing anticancer agents that can lead to enhance the therapeutic effect of radiation in cancer treatment. The aim of this study is to demonstrate the proof of this concept. THP-1 labeled with Qdot 800 or iron oxide (IO) effectively migrated into tumors of subcutaneous mouse model and increased recruitment after ionizing radiation. Functionalized liposomes carrying a radiosensitizing anticancer agent, doxorubicin, are successfully loaded in THP-1 (THP-1-LP-Dox) with reduced cytotoxicity, and THP-1-LP-Dox also was observed in tumors after intravenous administration. Here, we report that monocytes/macrophages as a biocarrier can be used as a selective tool for amplification of the therapeutic effects on radiotherapy for human cancer treatment.
\end{abstract}

\section{Introduction}

Nearly half of all cancer patients receive radiotherapy, either alone or in combination with other treatment modalities such as surgery or chemotherapy [1]. Although radiotherapy alone or with other treatments reaches a successive survival rates in treated patients with some cancers (e.g., early stage larynx cancer and non-small-cell lung cancer), for many other sites (e.g., glioblastomas, sarcomas, and advanced non-small-cell lung cancer), it shows low survival rates or the other chronic health problems such as second primary cancers and aggressive metastatic cancers to other organs $[1,2]$.
One of the reasons which reduce or neglect the therapeutic effect of radiotherapy is hypoxia in solid tumors. Hypoxia is a common feature in solid tumors and implicated in resistance to ionizing radiation and chemotherapy through multiple mechanisms caused by genetic or metabolic alterations in cancer cells [3]. Hypoxic tumor cells are significantly less responsive to radiotherapy and chemotherapy than well-oxygenated counterparts because of reduction or absence of the oxygen-derived free radicals that are needed to enhance DNA damage induced by ionizing radiation and nonproliferative hypoxic cells. The effect of these resistances brings increased invasiveness and metastatic potential, loss of apoptosis, and chaotic angiogenesis [4]. 
The accumulation of monocytes/macrophages from the blood circulation is a hallmark feature of cancers. As a result of release of a bunch of chemoattractants from altered tumor cells, monocytes are recruited into malignant tumors and differentiate into tumor-associated macrophages (TAMs) that facilitate tumor growth and survival [5]. The level of TAM numbers often correlates with aggressiveness and poor prognosis in human tumors and, in particular, appears to be affected by hypoxia [5]. TAMs are recruited to hypoxic sites by the release of macrophage chemoattractants (e.g., VEGF, endothelin-2, and EMAPLL) from hypoxic cells or by direct inhibition of the modality of TAMs [4]. These recruited and entrapped TAMs play an important role part in promoting tumor growth and progression by releasing many cytokines and growth factors such as VEGF and FGF2 [5].

The consequences of these complicated correlations among a limitation of radiotherapy, the presence of widespread hypoxia, and the accumulation of macrophages bring out a big hurdle in effective cancer treatments. Despite having difficulties in selecting a target for a cancer therapy, we focused on monocytes/macrophages that predominantly accumulate into tumors that resulted from radiotherapy and hypoxia. We postulated that monocytes/macrophages can be used as a biocarrier that loads anticancer agents to potentiate therapeutic effects based on cell-mediated drug delivery. Recently, one study reported a trial of the use of macrophages, acting as a Trojan Horse, in nanoparticle delivery and therapeutic system [6]. The efficient migration of Au-nanoshell laden macrophage toward T47D breast cancer spheroid and Au-nanoshell laden macrophage death induced by the near-infrared (NIR) irradiation in the hypoxic regions of a tumor spheroid was demonstrated.

The aim of this study is to verify our idea that monocytes/macrophages loading imaging or therapeutic agents can be used, either alone or in combination with radiotherapy, to treat cancer.

\section{Materials and Methods}

2.1. Preparation of THP-1. THP-1 (human acute monocytic leukemia cell line; ATCC, VA, USA) was cultured and maintained in RMPI 1640 medium (Gibco, NJ, USA) supplemented with 2-mercaptoethanol to a final concentration of $0.05 \mathrm{mM}, 10 \%(\mathrm{v} / \mathrm{v})$ heat-inactivated FBS plus 100 units $/ \mathrm{mL}$ penicillin and $100 \mu \mathrm{g} / \mathrm{mL}$ streptomycin. For experiments, THP-1 was pretreated with $20 \mathrm{nM}$ phorbol-12-myristate13-acetate (PMA; CalBiochem, Merk KGaA, Darmstadt, Germany) for 16 hrs.

2.2. Development of Mouse Models. A549 (ATCC, VA, USA) was cultured and maintained in F12K nutrient mixture (Kaighn's modification, Invitrogen, NY, USA) containing $10 \%$ FBS plus 100 units $/ \mathrm{mL}$ penicillin and $100 \mu \mathrm{g} / \mathrm{mL}$ streptomycin, respectively. Male Balb/c nude mice (5 weeks old) were purchased from SLC (Shizuoka, Japan) for generating subcutaneous mouse model. All experiments were performed following the protocol approved by Institutional Animal Care and Use Committee of ASAN Institute for Life
Science. $5 \times 10^{5}$ human non-small-cell lung cancer A549 cells in $100 \mu \mathrm{L}$ of PBS were injected into the subcutaneous tissue of the hind limb of each Balb/c nude mouse, after collecting cells by trypsinizing. When tumors grew to $100 \mathrm{~mm}^{3}$, mice were treated with THP-1 cell containing liposomal doxorubicin or free doxorubicin. For an irradiation (2 or $10 \mathrm{~Gy})$, tumors were irradiated using $6 \mathrm{MV}$ photon beam linear accelerator (CL/1800, Varian, CA).

2.3. Labeling THP-1 and Imaging. For labeling THP-1 cells, attached THP-1 on a plate was incubated with Qdot 800 (Qtracker 800; Molecular Probes, Inc., Eugene, OR) following the manufacturer's protocol. After labeling with Qdot $800,2 \times 10^{6}$ cells suspended in $200 \mu \mathrm{L}$ of PBS were injected through the tail vein of a xenograft mouse model bearing subcutaneous A549 tumor. In vivo optical imaging was taken using an IVIS spectrum imaging system (Caliper Life Science Inc.). The imaging was taken with a combination of a $430 \mathrm{~nm}$ excitation filter and a $780 \mathrm{~nm}$ emission filter. Gray scale photographic images and fluorescence color images were processed using living image V.3.2 (Caliper Life Science Inc.). Fluorescence signals are expressed as total flux (i.e., p/s).

THP-1 $\left(1 \times 10^{7}\right.$ cells per $10 \mathrm{~cm}$ dish $)$ was labeled with iron oxide (IO; $112 \mu \mathrm{g} / \mathrm{mL}$; Rezovist; Bayer AG, Zurich, Swiss) by incubation for $24 \mathrm{~h}$ at $37^{\circ} \mathrm{C}$ and $5 \% \mathrm{CO}_{2}$. After removing excessive IO by washing with PBS, THP-1 cells were collected by trypsinizing. In order to observe the migration of macrophages labeled with IO, $2 \times 10^{6}$ cells suspended in $200 \mu \mathrm{L}$ of PBS were injected through the tail vein of a xenograft mouse model bearing subcutaneous A549 tumor. MR images were taken prior to irradiation and 5 days after irradiation. The tumors were imaged with a 4.7-T Bruker Biospin imager (Bruker Medical Systems, Karlsruhe, Germany) prior to an injection for baseline and on days 2 and 5. All animals were anesthetized (1.5\% isoflurane in a $1: 2$ mixture of $\mathrm{O}_{2} / \mathrm{N}_{2} \mathrm{O}$ ) before imaging. The imaging protocol included a $\mathrm{T} 2 *$-weighted, gradient-echo sequence (repetition time msec/echo time msec, 356.5/10.3; flip angle, $30^{\circ}$ ). A transverse (orthogonal to tibia) section orientation was chosen for anatomic reproducibility of the image position and correlation with histological sections. The spatial resolution was $256 \times 256$ matrix; field of view, $2.18 \times 2.06 \mathrm{~cm}$; section thickness, $0.67 \mathrm{~mm}$; section gap, $0.33 \mathrm{~mm}$; number of sections, 16. All animals were scarified for histopathological evaluation after MR imaging.

2.4. Prussian Blue Staining. All animals were sacrificed by means of administration of inhalable pure $\mathrm{CO}_{2}$. The hind legs including a tumor were dissected, ex-articulated, fixed in $4 \%$ paraformaldehyde, and embedded in paraffin for staining. Paraffin sections of $5 \mu \mathrm{m}$ were prepared transversely. All slides were deparaffinized and rehydrated by sequenced sinking in xylene and ethanol. Prussian blue staining was performed using a mixture of $10 \%$ aqueous solution of potassium ferrocyanide and $20 \%$ aqueous solution of hydrochloric acid. After incubation for 20 minutes, the slides were washed at least 3 times in distilled water and counterstained with nuclear fast red for 5 minutes. After dehydration 


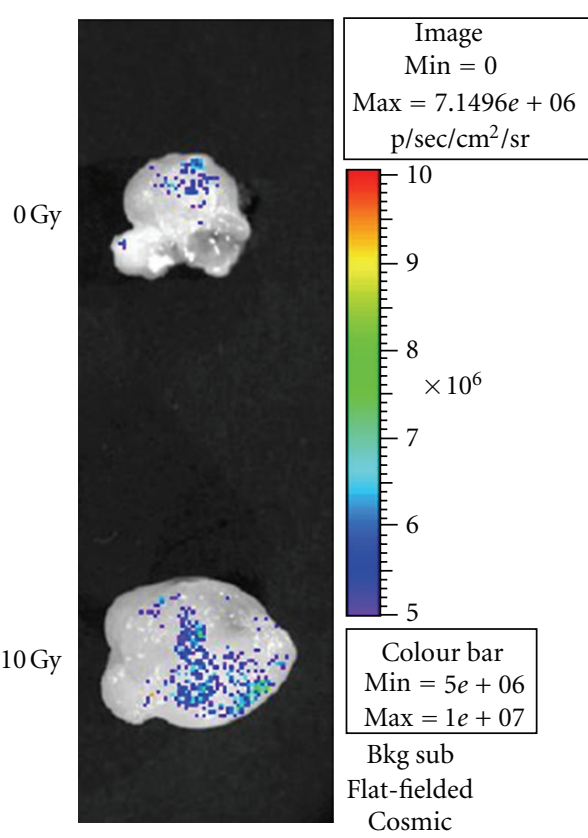

(a)

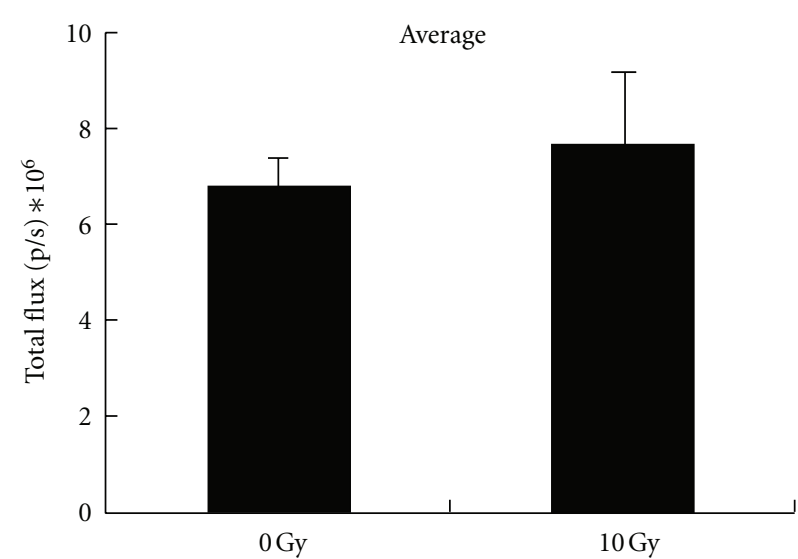

(b)

Figure 1: In vivo migration of THP-1-Qdot 800. IVIS images of A549 subcutaneous tumors after an injection of THP-1-Qdot 800 intravenously. (a) The fluorescence of Qdot was observed in tumors, and the intensity of fluorescence was increased after irradiation. (b) The total flux also shows slight increase after irradiation.

and clearing in ethanol and xylene, sections were evaluated using a microscope to determine the efficiency of labeling. Any cells that showed blue particles inside were considered labeled.

\subsection{Preparation and Characterization of Liposomal Dox-} orubicin (LP-Dox). Unilamellar liposomes of approximately $150 \mathrm{~nm}$ diameter were prepared by the extrusion method employing a laboratory extruder. Briefly, the lipid composition was based on 15:15:30:40 molar ratio of DPPC (dipalmitoylphosphatidylcholine, Nof Corporation, Tokyo, Japan): DPPE (dipalmitoylphosphatidylethanolamine, Sigma-Aldrich Co., MO, USA): DPPG-Na (dipalmitoylphosphatidylglycerol, Nof Corporation, Tokyo, Japan): Cholesterol (Wako Pure Chemical Industries. Ltd., Osaka, Japan). The lipids were dissolved and mixed in chloroform/methanol $(4: 1 \mathrm{v} / \mathrm{v})$ to assure a homogeneous mixture of lipids. The lipid film was thoroughly dried to remove residual organic solvent by placing the flask on a vacuum pump overnight. Then, the suspension obtained in $5 \mathrm{~mL}$ of hydration buffer ( $\mathrm{pH} 4.0,10 \mathrm{mM}$ of HEPES and $120 \mathrm{mM}$ Ammonium Sulfate) was extruded through polycarbonate filters with $800,400,200$, and twice of $100 \mathrm{~nm}$ pore size for a size control. The average concentration of liposome was $5 \mathrm{mg} / \mathrm{mL}$ calculated with a cholesterol quantitation kit (BioVision incorporated, CA, USA) following a manufacturer's protocol. Encapsulation of doxorubicin hydrochloride (doxorubicin; Dox; Sigma-Aldrich, MO, USA) into the liposomal core was performed at $60^{\circ} \mathrm{C}$ in a water bath for $30 \mathrm{~min}$ after the $\mathrm{pH}$ exchange of buffer $(\mathrm{pH}$ 7.4,
$10 \mathrm{mM}$ of HEPES, $100 \mathrm{mM}$ Sodium chloride, and $100 \mathrm{mM}$ Sucrose) in Sephadex G25 column (GE Healthcare Life Sciences, NJ, USA). The ratio of added doxorubicin to liposome is $1.8 \mathrm{mg}: 15 \mathrm{mg}$. Removal of nonencapsulated doxorubicin was achieved by size exclusion chromatography using Sephadex G25. The encapsulate doxorubicin concentration was determined by a measurement of the fluorescence intensity (excitation at $537 \mathrm{~nm}$ and emission at $584 \mathrm{~nm}$ ) with an Enspire 2300 multilabel reader (Perkin Elmer, MA, USA). The average concentration of doxorubicin in LP-Dox was $0.6 \mathrm{mg} / \mathrm{mL}$. For the size determination of liposome, a Zetasizer (Nano-ZS, Malvern instruments, Malvern, UK) was used.

2.6. THP-1-LP-Dox Viability and Uptake. $5 \times 10^{3}$ THP-1 cells in $100 \mu \mathrm{L}$ medium were plated in each well of a 96well plate. Then, Dox or LP-Dox was added by concentration gradients, $1,2,10$, or $20 \mu \mathrm{g} / \mathrm{mL}$. Macrophages with Dox or LP-Dox were additionally incubated for 3, 6, or 24 hours. At the end of incubation time point, Dox or LP-Dox was removed by medium changing and washing. For a viability assay, macrophages were incubated with $100 \mu \mathrm{L}$ of fresh medium containing $10 \mu \mathrm{L}$ of the CCK8 solution (Dojindo Laboratories, Kumamoto, Japan) for 3 hours at $37^{\circ} \mathrm{C}$ in $5 \%$ $\mathrm{CO}_{2}$. The absorbance was measured at $450 \mathrm{~nm}$ using an Enspire 2300 multilabel reader (Perkin Elmer).

To determine the concentration of Dox uptaken by macrophages, the survived cells were lysed at the end point of an incubation time, mixed with methanol to melt liposomes, and then measured fluorescence intensity. 


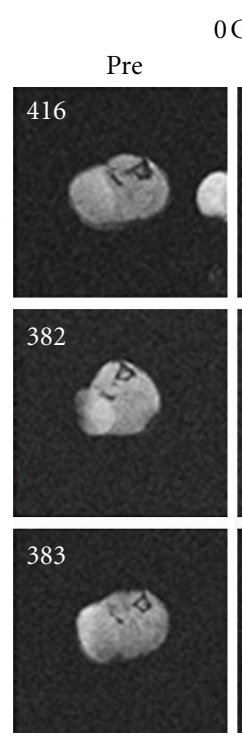

Post
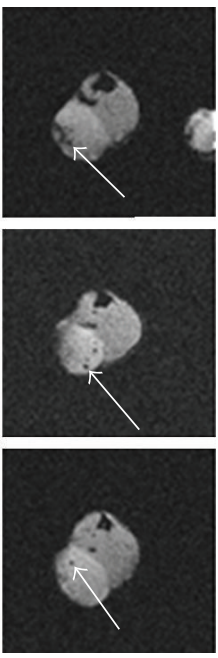

Gy
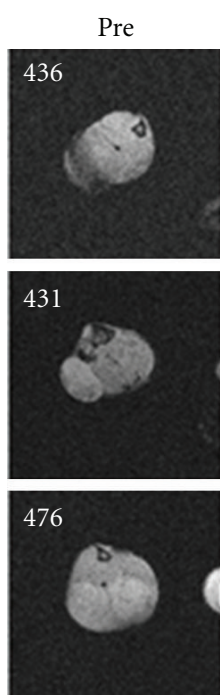

Post
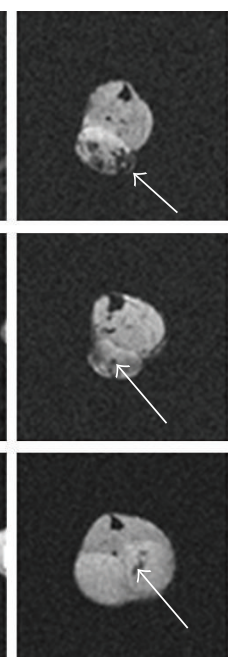
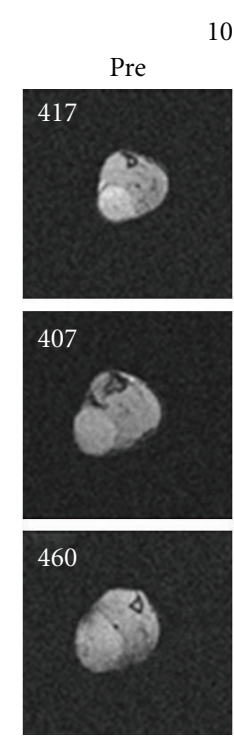

$10 \mathrm{~Gy}$

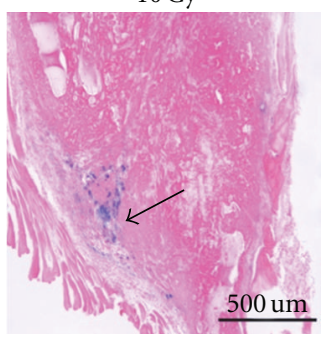

(b)

2 Gy
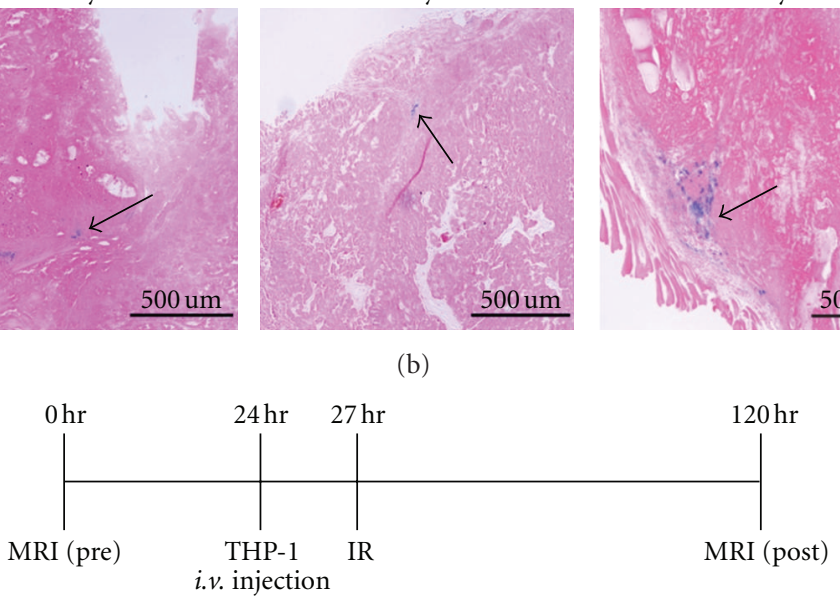

(c)
Post
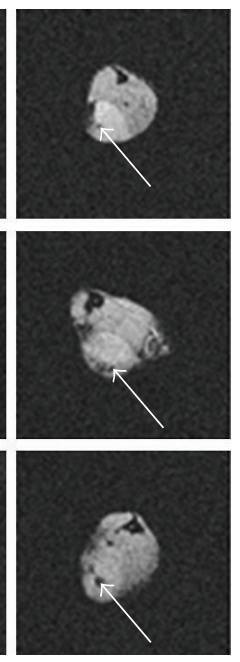

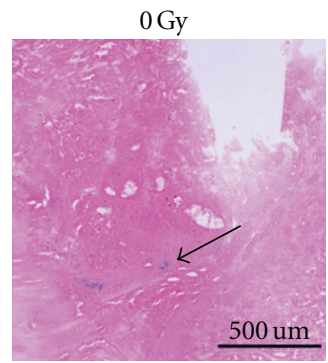

Figure 2: In vivo migration of THP-1-IO. MR images of A549 subcutaneous tumors after an injection of THP-1-IO intravenously. (a) Tumors contained IO, and the volume of IO detected was gradually increased followed with an increasing dose of irradiation. Numbers indicate individual samples. (b) IO was detected by Prussian blue staining (arrows) in a tumor from mice injected THP-IO. (c) The time frame indicates MR imaging schedule. Calibration bar $=500 \mu \mathrm{m}$.

2.7. THP-1-LP-Dox Imaging. The plate-adherent THP-1 cells (approximately $5 \times 10^{6}$ cells per a $6 \mathrm{~cm}$ dish) were incubated with LP-Dox containing $20 \mu \mathrm{g} / \mathrm{mL}$ of Dox for 6 hours. After removing the remained LP-Dox in medium by washing with PBS, live THP-1 was harvested and counted. To determine the concentration of Dox uptaken by THP-1, $5 \times 10^{6}$ cells were lysed, mixed with methanol to melt liposomes, and then measured fluorescence intensity. For a test of delivery of macrophages-LP-Dox in subcutaneous or metastasis mouse models, $60 \mu \mathrm{g}$ of Dox loaded THP-1-LP-Dox (approximately $10^{6}$ cells) in $100 \mu \mathrm{L}$ of PBS was administrated into a mouse through the tail vein, when a tumor volume was reached at $100 \mathrm{~mm}^{3}$ in a subcutaneous model. Tumors were collected at 24 hours postinjection and subjected to fluorescence microscopic analysis. In order to collect tumors from each mouse at the end point of an experiment, mice were euthanized with $\mathrm{CO}_{2}$ inhalation. Tumors collected were fixed in $4 \%$ paraformaldehyde and embedded in paraffin for staining. Paraffin sections of $5 \mu \mathrm{m}$ were prepared and stained with DAPI (VECTASHIELD Mounting Media with DAPI, Vector Laboratories, Inc., CA) after deparaffinization and dehydration. Images were captured and processed with a fluorescence microscope (Bx53; Olympus, Tokyo, Japan) equipped with a cooled CCD camera (ORCA-R2; Hamamtsu Photonics K.K., Hamamatsu, Japan).

2.8. Statistical Analysis. All data expressed as mean $\pm \mathrm{SD}$ are representative of at least three different experiments. Comparisons between individual points were performed by a Student's $t$-test using Prism statistical software (GraphPad, 


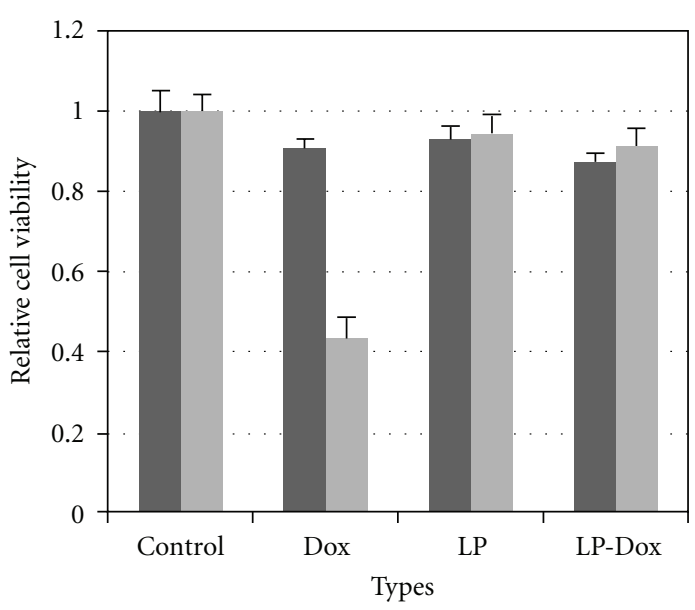

$1 \mu \mathrm{g} / \mathrm{mL}$

$2 \mu \mathrm{g} / \mathrm{mL}$

(a)

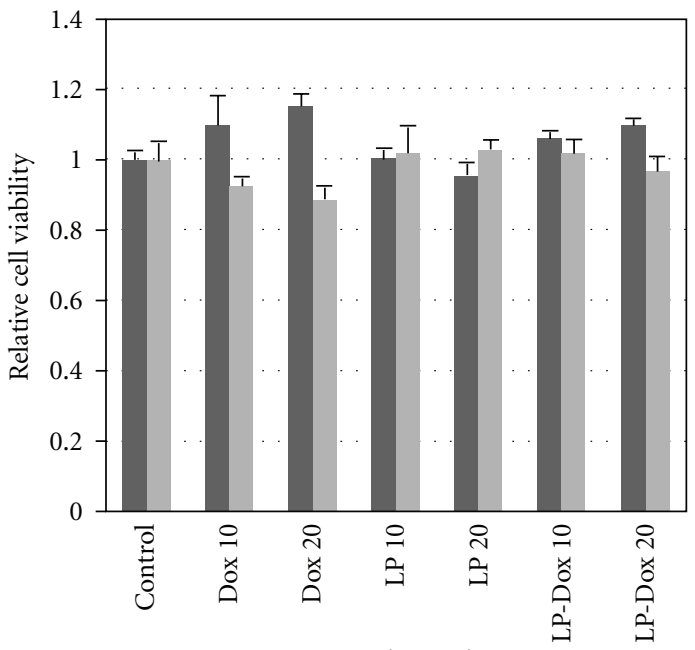

Types $(\mu \mathrm{g} / \mathrm{mL})$

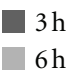

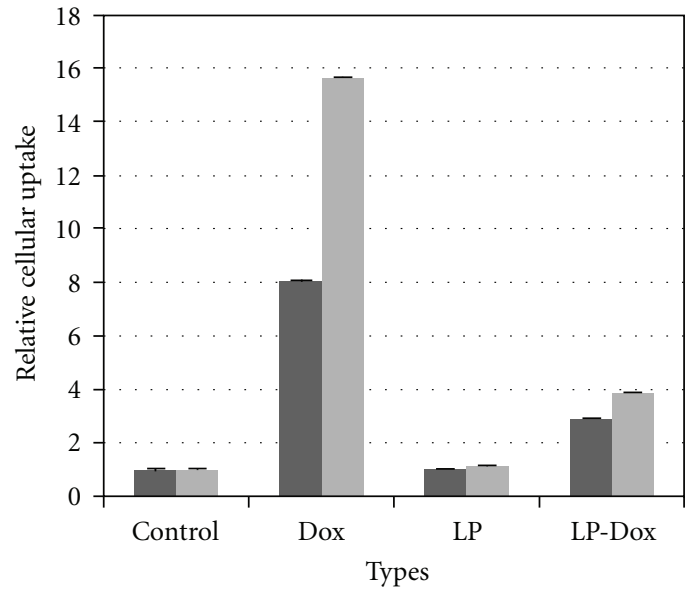

$1 \mu \mathrm{g} / \mathrm{mL}$

$2 \mu \mathrm{g} / \mathrm{mL}$

(b)

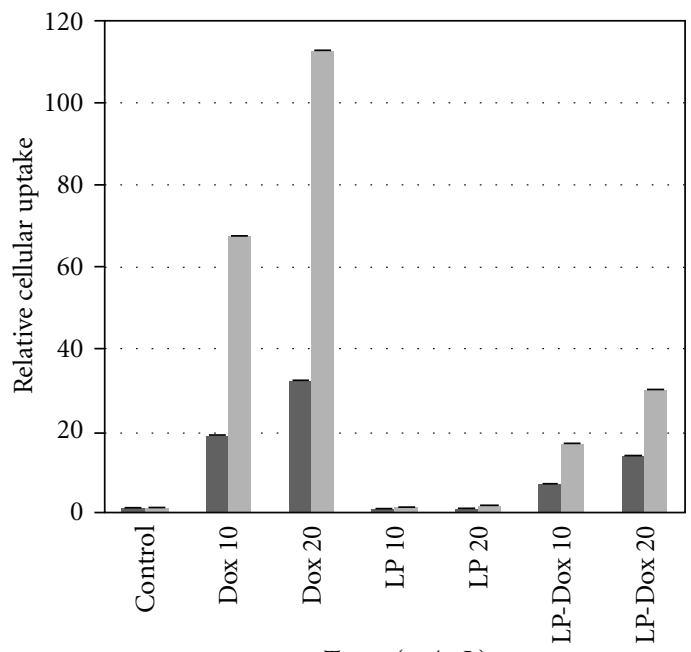

Types $(\mu \mathrm{g} / \mathrm{mL})$

$3 \mathrm{~h}$

$6 \mathrm{~h}$

(c)

(d)

FIgure 3: Conditions for incubating THP-1 with LP-Dox. (a) THP-1 viability after $24 \mathrm{~h}$ incubation of Dox, LP, or LP-Dox. (b) The uptake volume of Dox in THP-1 after $24 \mathrm{~h}$ incubation of Dox, LP, or LP-Dox. (c) THP-1 viability after 3 or $6 \mathrm{~h}$ incubation of Dox, LP, or LP-Dox. (d) The uptake volume of Dox in THP-1 after 3 or $6 \mathrm{~h}$ incubation of Dox, LP, or LP-Dox.

CA, USA). $P$ values less than 0.05 were considered statistically significant.

\section{Results}

3.1. In Vivo Migration of THP-1 Labeled with Qdot 800 in a Xenograft Mouse Model. In order to demonstrate our hypothesis, we first needed to verify the migration ability of macrophages toward tumors in vivo. We tested the in vivo migration and infiltration ability of THP-1 using a xenograft mouse model bearing a subcutaneous A549 tumor. For long-term tracking or in vivo imaging easily, THP1 cells were labeled with Qdot 800 which supports the intense fluorescence under various biological conditions and less autofluorescence in tissues. After 5 days postinjection of labeled THP-1 cells through tail vein, we imaged tumors with IVIS spectrum imaging system. Fluorescence located in tumors was apparently observed (Figure 1(a)). In order to observe the radiation effect on the migration of macrophages, we irradiated tumors with $10 \mathrm{~Gy}$ after THP1 injection. Interestingly, we observed increased intensity of fluorescence in tumors after $10 \mathrm{~Gy}$ of radiation (Figures 1(a) and $1(\mathrm{~b}))$, compared to nonirradiated tumors.

\subsection{In Vivo Migration of THP-1 Labeled with IO in a Xenograft} Mouse Model. In addition to Qdot, we also attempted to 

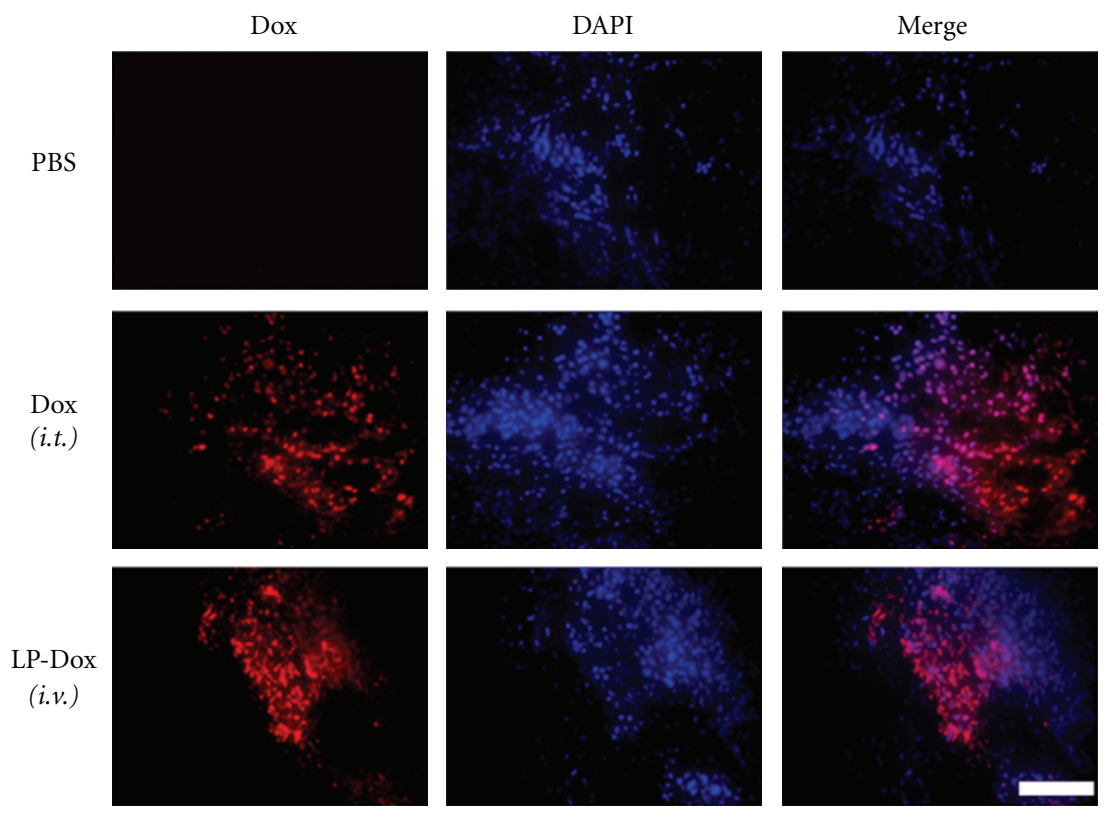

FIgURE 4: Distinct fluorescent expressions of Dox or LP-Dox (red) in tumors at $24 \mathrm{~h}$ postinjection of THP-1-LP-Dox. DAPI (blue) was used for a counter staining. Calibration bar $=50 \mu \mathrm{m}$.

label THP-1 cells with IO to expand to other labeling methods. Like results with Qdots, distinct IO expressions in tumors after intravenous (i.v.) injection were observed in MR imaging and the expression was enhanced after radiation (Figure 2(a)). We confirmed this result with blue stained IO in tumors with a Prussian blue staining (Figure 2(b)). We observed a large number of blue stained IO spread in a deep side of a tumor from a mouse injected macrophages IO (Figure 2(b)). We gave radiation with different doses, 2 and $10 \mathrm{~Gy}$, if the radiation dose affects the migration ability of macrophages. Interestingly, we observed increased intensity of IO expression gradually followed with increased dose of radiation (Figures $2(\mathrm{a})$ and $2(\mathrm{~b})$ ).

These results led to open our hypothesis that macrophage can be used as a biocarrier, such as imaging or anticancer agents, to deliver into tumors with assistance of radiation or not.

3.3. Development of THP-1-LP-Dox. Next, we asked to approach the way to load anticancer agents to THP-1 cells. The majority of anticancer agents has overt cytotoxicity. In order to use macrophages as a biocarrier for an anticancer agent, we needed to protect macrophages from a direct toxicity of a selected agent, Dox, as a cargo until they can reach tumors alive. We chose liposomes for encapsulating drugs to release slowly. Liposomes, nature biomolecules, have the ability to carry a greater amount of drugs while minimizing the risk associated with premature leakage. Dox was chosen as an anticancer drug in our experiment because of its unique fluorescence characteristic in excitation at $537 \mathrm{~nm}$ and emission at $584 \mathrm{~nm}$ which has the easy accesses to monitor their action. We successfully encapsulated Dox with liposome (approximately $150 \mathrm{~nm}$ diameter) composed of DPPC:DPPE:DPPG-Na:Cholesterol $=15: 15: 30: 40$ molar ratio followed by our regular protocol.

We then tested a toxicity of LP-Dox to THP-1 by CCK8 assay compared with free Dox as a control. With relatively long-term incubation $(24 \mathrm{~h})$, LP-Dox showed almost no cytotoxicity in our given concentrations compared to free Dox (Figure 3(a)), although the total volume of cellular uptake of LP-Dox was much less than free Dox, which was not enough to our expectation (Figure 3(b)). Therefore, we multiplied a concentration of LP-Dox into cells and reduced an incubation time to increase loading efficacy of LP-Dox in cells without any cytotoxicity. With short-time incubations ( 3 and $6 \mathrm{~h}$ ), we observed only slight cytotoxicity in free Dox with a high concentration (Figure 3(c)). The total volume of cellular uptake of LP-Dox was increased with the 10 times higher concentration as we added $(20 \mu \mathrm{g} / \mathrm{mL})$ without any effects on cell viability (Figure $3(\mathrm{~d})$ ). We chose this concentration of LP-Dox $(20 \mu \mathrm{g} / \mathrm{mL})$ and an incubation time $(6 \mathrm{~h})$ for conditions of the next in vivo experiment.

\subsection{In Vivo Migration of THP-1-LP-Dox in a Xenograft Mouse Model. Although we previously observed the migration by using THP-1 labeled with Qdot or IO toward tumor site in vivo, we needed to confirm the migration of THP-1- LP-Dox into the tumor site in vivo. With an established subcutaneous mouse model that developed A549 tumor on the right thigh, we attempted to verify the migration of THP- 1-LP-Dox after i.v. injection through tail vein. We collected tumors from each mouse at 24 hours postinjection of THP-1-LP-Dox and observed fluorescence on tumor tissues after DAPI counterstaining. Compared with a tumor which showed widespread Dox expression in the tumor after an intratumoral (i.t.) injection of free Dox, we observed similar}


amount of Dox expression infiltrated in the tumor from the group of THP-1-LP-Dox (Figure 4). Unlike free Dox, Dox in these tumors was observed in the cells between tumor cells. These results indicated that macrophages effectively carried LP-Dox into the tumor site and could be used as a valuable tool for carrying anticancer agents.

\section{Discussion}

This study is to prove the concept of our idea that macrophages/monocytes can be used for a valuable tool for drug delivery into specific tumor sites. The unique characteristics of macrophages offered their potential as a target-specific drug carrier. Macrophages are immunocytes that migrate across the blood-tumor barriers to the tumor and can be collected easily from the blood. And their innate phagocytic capability provides an easy access of loading with therapeutic nanoparticles.

As a first trial in this study, we used THP-1 cells, which are originated from human acute monocytic leukemia cells, for a drug carrier. Although these cells loading with imaging or anticancer agents showed an active targeting and migrating ability towards tumors in our study, they should be considered more carefully as a carrier. THP- 1 cells are cancer cells which can bring a chance of the second tumorigenesis and an immune rejection reaction in the body. Our final goal of the study is engaged on applying autoimplantation of monocytes/macrophages isolated from a cancer patient, as a biocarrier for chemotherapeutic anticancer agents not only to improve their therapeutic efficacy but also to eliminate an immune rejection response.

Recently, several studies using human or mouse macrophages as a delivery for nanoparticles or viruses have been reported [6-9]. Notably, macrophages were served as a "Trojan Horse" delivery vector for nanoparticle therapeutics into inaccessible tumor regions, such as a hypoxic area. Choi et al. [6] demonstrated a study of using Trojan Horse nanoparticle delivery and therapeutics within their several critical steps that are the efficient phagocytosis of gold nanoshells by macrophages, photoinduced ablation of gold nanoshells laden macrophages, tumor recruitment, and photoinduced cell death of a human breast tumor spheroid. In another study, similar to our study, macrophages carrying 5-fluorouracil (5-FU) encapsulated in oligomannosecoated liposomes (OML) were used for drug delivery in a mouse intraperitoneal (i.p.) metastasis model [7]. The controlled tumor development by coadministration of OMLencased 5-FU and OML-encased magnetic nanoparticles, followed by treatment with an alternating magnetic field, was demonstrated in their study. Although these works were well demonstrated macrophages as a biocarrier, it was not proved whether the in vivo systemic approach was accessible.

Cell-mediated drug delivery using immunocytes, mononuclear phagocytes, and stem cells, and so forth offers targeted drug transfer to specific tumor sites with reduced drug immunogenicity and cytotoxicity [10]. Despite the advantages, this field of study is still considered an unexplored area with several limitations including high drug loading volume in cells to be enough to affect tumor cell death, active migration ability and time-controlled drug release at the desirable site without any leakage of drugs, and the improvement of safe and relatively stable drug formulation to be loaded in cells.

In this study, we showed the effective migration of THP1 loading imaging agents or anticancer agents encapsulating liposomes into tumor sites in vivo. Also, increased accumulation of THP-1 cells in tumors was observed after radiation. These results suggest macrophage-mediated liposome-encapsulated drug delivery system alone or with a combination with a radiation is a new potential therapeutic system to amplify an efficacy in cancer treatment. However, in order to approach this, there are many views to be demonstrated further; when drug release is performed at the right sites in the body, how the drug loading volume in cells can be increased with any toxicity, how can we improve more sophisticated time-control release of drugs into the desired sites, and there is an efficient and obvious therapeutic effect we can recognize after a treatment.

\section{Conflict of Interest}

The authors declare no conflict of interest.

\section{Acknowledgments}

This work was supported by a grant of the Nuclear R\&D program through the Korea Science and Engineering Foundation funded by the Ministry of Education, Science and Technology of Korea (2008-03876), the Korean Health Technology R\&D Project, Ministry for Health and Welfare, Republic of Korea (A062254 and A102059), and the Basic Science Research Program through the National Research Foundation of Korea (NRF) funded by the Ministry of Education, Science and Technology (KRF-2008-313E00444).

\section{References}

[1] A. C. Begg, F. A. Stewart, and C. Vens, "Strategies to improve radiotherapy with targeted drugs," Nature Reviews Cancer, vol. 11, no. 4, pp. 239-253, 2011.

[2] W. D. Newhauser and M. Durante, "Assessing the risk of second malignancies after modern radiotherapy," Nature Reviews Cancer, vol. 11, no. 6, pp. 438-448, 2011.

[3] W. R. Wilson and M. P. Hay, "Targeting hypoxia in cancer therapy," Nature Reviews Cancer, vol. 11, no. 6, pp. 393-410, 2011.

[4] C. Murdoch and C. E. Lewis, "Macrophage migration and gene expression in response to tumor hypoxia," International Journal of Cancer, vol. 117, no. 5, pp. 701-708, 2005.

[5] C. Lewis and C. Murdoch, "Macrophage responses to hypoxia: implications for tumor progression and anti-cancer therapies," American Journal of Pathology, vol. 167, no. 3, pp. 627635, 2005.

[6] M. R. Choi, K. J. Stanton-Maxey, J. K. Stanley et al., "A cellular trojan horse for delivery of therapeutic nanoparticles into tumors," Nano Letters, vol. 7, no. 12, pp. 3759-3765, 2007.

[7] Y. Ikehara, T. Niwa, L. Biao et al., "A carbohydrate recognitionbased drug delivery and controlled release system using 
intraperitoneal macrophages as a cellular vehicle," Cancer Research, vol. 66, no. 17, pp. 8740-8748, 2006.

[8] R. F. Bressani, A. S. Nowacek, and S. Singh, "Pharmacotoxicology of monocyte-macrophage nanoformulated antiretroviral drug uptake and carriage," Nanotoxicology, vol. 5, pp. 592-605, 2011.

[9] S. J. Madsen, S. K. Baek, A. R. Makkouk, T. Krasieva, and H. Hirschberg, "Macrophages as cell-based delivery systems for nanoshells in photothermal therapy," Annals of Biomedical Engineering, vol. 40, no. 2, pp. 507-515, 2012.

[10] E. V. Batrakova, H. E. Gendelman, and A. V. Kabanov, "Cellmediated drug delivery," Expert Opinion on Drug Delivery, vol. 8, no. 4, pp. 415-433, 2011. 

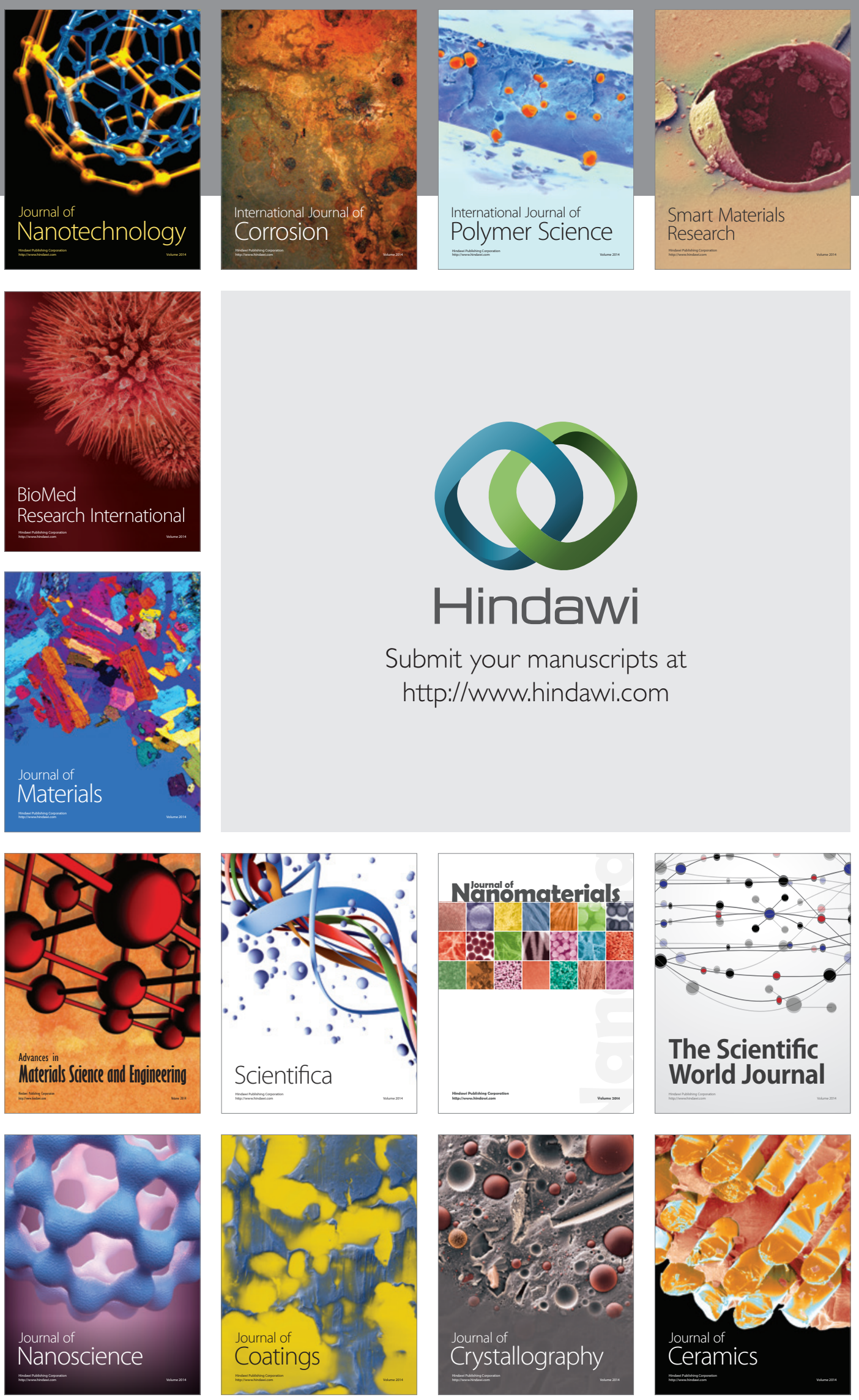

The Scientific World Journal

Submit your manuscripts at

http://www.hindawi.com

\section{World Journal}

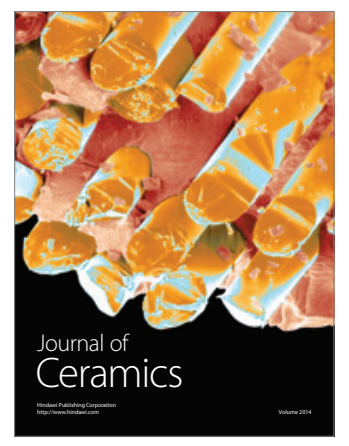

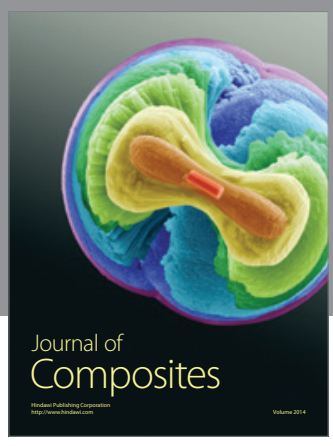
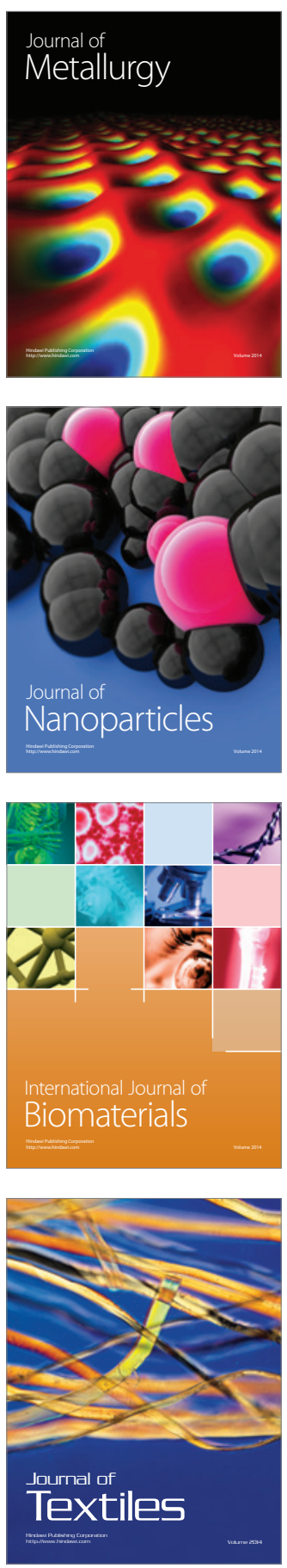